\title{
Un «oui» net en faveur de l'Assemblée des délégués
}

\author{
Lors de sa séance au Landhaus de Soleure, la Conférence des présidents a voté sa propre dissolution
}

Lü. Une FMH allégée, plus souple et à l'efficacité accrue et, finalement, un corps médical plus fort: tels sont les principaux objectifs visés par la réforme interne des structures en cours. Après la journée de travail organisée au «Stade de Suisse» pour connaître l'avis des membres de la Chambre médicale et la consultation à grande échelle qui a suivi, la Conférence des présidents tenue à Soleure a maintenant pris des décisions fondamentales, claires et porteuses d'avenir.

En ouverture de séance, Jacques de Haller, président de la FMH, a précisé aux nombreux participants qu'il s'agissait d'élaborer un modèle pour des statuts à l'attention de la Chambre médicale (ChM) de début décembre. Cet objectif a été atteint: en fin de journée, les synthèses découlant de la consultation réalisée auprès des sociétés médicales ont pratiquement toutes été approuvées à une forte majorité.

\section{Assemblée des délégués au lieu de Conférence des présidents}

Par un oui clair, les présidents présents ont accepté que leur organe - la Conférence des présidents ou $\mathrm{CP}$ - soit remplacé par une Assemblée des délégués (AD). Mais ils ont manifesté tout aussi clairement leur volonté de laisser à la ChM sa fonction de contrôle en lui octroyant le droit de recourir contre les décisions de l'AD. En revanche, ils ont refusé d'accorder à la ChM un droit de regard sur les membres de l'AD.
La composition de l'AD a suscité des discussions nourries quant au nombre de délégués, qui s'est vu fixé provisoirement entre 25 et 32 . Il reviendra à la ChM de préciser ce chiffre. Les sièges seront répartis selon un système fixe sous la forme proposée.

\section{Un seul président}

Le deuxième point principal controversé de l'ordre de jour portait sur la question suivante: la FMH doit-elle continuer à se doter d'un seul président ou faut-il que la ChM et la future $\mathrm{AD}$ aient chacune leur propre présidence? Ici aussi, les délégués à la $\mathrm{CP}$ se sont clairement prononcés en faveur de la «sveltesse», c'est-à-dire d'un seul président.

Une limitation de la durée de fonction des membres du Comité central (CC) et la création d'une Commission de gestion (CdG) étaient des points incontestés au cours de la consultation déjà. Enfin, les présidents ont estimé que les nouveaux statuts devront prévoir une incompatibilité entre un mandat au CC ou à la CdG et une responsabilité dans une entreprise commerciale active dans le domaine de la santé.

Toutes ces décisions de la CP vont maintenant être traduites concrètement dans un nouveau projet de statuts qui, lui, fera l'objet d'une discussion approfondie lors de la Chambre médicale extraordinaire du 2 décembre prochain. 